\title{
Plasma amino acid concentration in the hepatic vein compared to peripheral blood
}

\begin{abstract}
Introduction: Patients with liver disease show abnormalities in the plasma aminoacid profile. In order to investigate and further advance in the study of protein and amino acid metabolism in liver cirrhosis we studied the amino acid differences between hepatic vein and peripheral blood.
\end{abstract}

Methods: We studied 13 male adult patients with biopsy proven liver cirrhosis and endoscopic evidence of portal hypertension. Patients were fasted for at least 6hours before the study. Hepatic vein samples were obtained from a catheter placed in the hepatic vein and peripheral blood samples were obtained from the femoral vein.

Results: We observed that in most cases there was a lower concentration of the individual amino acids in the hepatic vein compared to those in the peripheral vein but they were not significant except in the case of alanine.

Conclusion: We confirmed the characteristic pattern of increased concentration of the aromatic amino-acids (AAA) and decreased concentration of branched-chain amino acids (BCAA). The general trend towards lesser concentration of amino acids in the hepatic vein compared to peripheral vein observed in this study probably reflects the combined effect of amino acid exchange across the whole splanchnic bed. The higher alanine concentration in peripheral blood, in this case representing blood coming from muscles, seems to be the result of the glucose-alanine cycle.

Keywords: amino acids, cirrhosis, liver, hepatic vein, AAA, BCAA

\author{
Volume 4 Issue I - 2018 \\ Octavio Campollo,' Massimo Graffeo, ${ }^{2}$ Aiden \\ McCormick ${ }^{3}$ \\ 'Center of studies on alcohol and addictions, Antiguo Hospital \\ Civil de Guadalajara, Mexico \\ ${ }^{2}$ Department of endoscopic gastroenterology, Liver and \\ ultrasound unit, Italy \\ ${ }^{3}$ St.Vincent's University Hospital, Ireland
} alcohol and addictions, Antiguo Hospital Civil de Guadalajara, Calle Hospital 278, Col. El Retiro.Guadalajara, Jal, CP 44280, Mexico,Email calcohol@hotmail.com

Received: January 10, 2018 | Published: January 30, 2018

\section{Introduction}

The liver plays a key role in the metabolism of amino acids as it removes releases and incorporates them into different proteins and compounds. Patients with liver disease show abnormalities in the plasma amino-acid profile. ${ }^{1-3}$ In advanced liver disease there is usually an increased concentration of the aromatic amino-acids (AAA) tyrosine, phenylalanine and tryptophan, and decreased concentration of branched-chain amino acids (BCAA) leucine, isoleucine and valine in plasma. ${ }^{4-12}$ The methods used to investigate amino acid metabolism have varied widely i.e. basal measurements, protein challenge tests, fasting and post-absorptive period, samples obtained from peripheral or arterial blood to mention a few., ${ }^{3,10}$

A different approach in the study of amino acid metabolism is the measurement of amino acid concentrations in the liver (hepatic vein) and compares it with that from peripheral blood. Nevertheless, there is not much information about the hepatic vein-peripheral venous differences in plasma amino acids in cirrhotic patients. ${ }^{13}$ That information may yield important information about protein and amino acid metabolism in patients with liver cirrhosis. In this study we investigated the hepatic vein-peripheral venous blood differences in plasma amino acid concentrations using the method of direct sampling of the hepatic vein simultaneously with a peripheral vein.

\section{Methods}

\section{Study subjects}

We studied male adult patients with biopsy proven liver cirrhosis and endoscopic evidence of portal hypertension i.e. oesophageal varices. Most of them had history of gastrointestinal bleeding. The severity of liver disease varied. The patients were being studied in a protocol to investigate haemodynamic changes in the portal system in cirrhosis. The protocol was approved by the Ethics committee of the Royal Free Hospital and all patients gave written, informed consent to participate in the study.

\section{Procedures}

Patients were fasted for at least 6hours before the study. Blood samples were obtained at basal conditions. Hepatic vein samples were obtained from a catheter placed in the hepatic vein and peripheral blood samples were obtained from the femoral vein. In more detail the right femoral vein was cannulated using an $8 \mathrm{~F}$ introducer sheath. A 7F balloon tipped catheter (Cordins SA, Miami, USA) was introduced into the hepatic vein under fluoroscopic control. ${ }^{14}$ The position of the catheter and the adequacy of wedging were checked by injecting intravenous contrast into the hepatic vein with the balloon inflated. Blood samples from the two sites were obtained at more or less the same time with a maximum 1 minute difference between them. Blood samples were prepared for amino acid analysis as described previously. ${ }^{4}$

\section{Results}

In most cases there was a lower concentration of the individual amino acids in the hepatic vein compared to those in the peripheral vein (Figure 1) but they were not significant except in the case of alanine. 


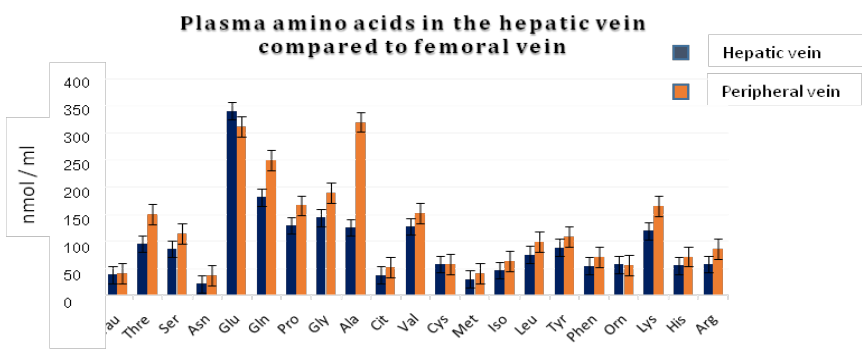

Figure I Plasma amino acids in the hepatic vein compared to femoral vein.

\section{Discussion}

Plasma amino acids in the postabsorptive state reflect the balance between release by extrahepatic tissue, primarily from muscle, and uptake by the liver. ${ }^{15}$ Following protein digestion after a meal BCAA are transferred from the gut through the liver to peripheral tissues; the other amino acids including the aromatic amino acids (AAA) and methionine are retained to a greater extent by the splanchnic area particularly by the liver. ${ }^{12,16,17}$ BCAA are metabolized in extrahepatic tissues i.e. skeletal muscle. ${ }^{18}$

A characteristic pattern of plasma amino acids has been described in cirrhotic subjects ${ }^{4-9,19,20}$ and metabolic and biochemical differences have been shown between stable and unstable cirrhotics. ${ }^{4}$ In this study we observed the characteristic pattern of increased concentration of the aromatic amino-acids (AAA) tyrosine, phenylalanine and tryptophan, and decreased concentration of branched-chain amino acids (BCAA) leucine, isoleucine and valine. ${ }^{4-12}$ We have previously reported differences between different stages of liver disease and small or no significant differences between patients with stable liver disease and normal subjects. ${ }^{4}$ The general trend towards lesser concentration of amino acids in the hepatic vein compared to peripheral vein observed in this study probably reflects the combined effect of amino acid exchange across the whole splanchnic bed. The peripheral blood amino acid concentration was very similar to what we have previously reported. ${ }^{20}$ In this study, however, we observed a higher concentration of peripheral blood alanine compared to that in the hepatic vein which is probably the result of the "glucose-alanine cycle". Muscle provides precursors for hepatic glucose synthesis by releasing gluconeogenic amino acids particularly during fasting. Insulin inhibits muscle protein breakdown and the release of amino acids. As insulin levels decrease in fasting, skeletal muscle releases amino acids which are carried to the liver for gluconeogenesis. ${ }^{21}$ In a normal subject in the postabsorptive state, there is a net release of amino acids from muscle, the output of alanine and glutamine exceeding that of all other amino acids. The amount of alanine and glutamine taken up by the liver upper intestine, pancreas and spleen (splanchninc bed) appeared to correspond fairly closely to the amounts released by muscle. ${ }^{13}$ The higher alanine concentration in peripheral blood, in this case representing blood coming from muscles, seems to be the result of the glucose-alanine cycle. There were smaller increases in the levels of tyrosine and phenylalanine in peripheral blood.

\section{Limitations}

In this study it was not possible to measure uptake and release rates because we did not measure blood flows nor did we measure other important metabolites because the patients were being assessed haemodinamically in order to decide which therapeutic modality was best to control chronic recurrent variceal bleeding (e.g. endoscopic, pharmacologic or surgery). Future studies should include blood flow measurements or use of tracers to solve that problem. Another possibility would be to administer food or formulae to investigate changes in the pattern of amino acid profile across the splanchnic bed.

\section{Acknowledgements}

We thank Prof. Neil McIntyre and Prof. Andrew K Burroughs for their support and guidance and Ms. Angela Madden for her nutrition supervision.

\section{Conflict of interest}

The authors declare no conflict of interest.

\section{References}

1. Tavill AS. Protein metabolism and the liver. In: Wright R, editors. Liver and biliary disease. London: Saunders; 1985. p. 87-113.

2. Fischer JE, Rosen HM, Ebid AM, et al. The effect of normalization of plasma amino acids on hepatic encephalopathy in man. Surgery. 1976;80(1):77-91.

3. Marchesini G, Bianchi GP, Vilstrup H, et al. Plasma clearences of branched-chain amino acids in control subjects and in patients with cirrhosis. J Hepatol. 1987;4(1):108-117.

4. Campollo O, Sprengers D, McIntyre N. The BCAA/AAA ratio of plasma amino acids in three different groups of cirrhotics. Rev Invest Clin. 1992;44(4):513-518.

5. Schulte-Frohlinde E, Wagenpfeil S, Willis J, et al. Role of meal carbohydrate content for the imbalance of plasma amino acids in patients with livber cirrhosis. J Gastroenterol Hepatol. 2007;22(8):1241-1248.

6. Silk DB. Branched-chain amino acids in liver disease: fact or fantasy? Gut. 1986;27(Suppl 1):103-110.

7. Ganda OP, Ruderman NB. Muscle nitrogen metabolism in chronic hepatic insufficiency. Metabolism. 1976;25(4):427-435.

8. Schauder P, Schroder K, Herbertz L, et al. Evidence for valine intolerance in patients with cirrhosis. Hepatology. 1984;4(4):667-670.

9. Mullen KD, Denne SC, McCullough AJ, et al. Leucine metabolism in stable cirrhosis. Hepatology. 1986;6(4):622-630.

10. Marchesini G, Bianchi G, Zoli M, et al. Plasma amino acid response to protein ingestion in patients with liver cirrhosis. Gastroenterology. 1983;85(2):283-290.

11. Montanari A, Simoni I, Vallisa D, et al. Free amino acids in plasma and skeletal muscle of patients with liver cirrhosis. Hepatology. 1988;8(5):1034-1039.

12. Tomiya $T$, Omata M, Fujiwara K. Branched-chain amino acids, hepatocyte growth factor and protein production in the liver. Hepatol Res. 2004;30S:S14-S18.

13. Wahren J, Felig P, Hagenfeldt L. Effect of protein ingestion on splanchnic and leg metabolism in normal man and in patients with diabetes mellitus. J Clin Invest. 1976;57(4):987-999.

14. McCormick PA, Dick R, Graffeo M, et al. The effect of non-protein liquid meals on the hepatic venous pressure gradient in patients with cirrhosis. J Hepatol. 1990;11(2):221-225.

15. Bianchi G, Marzocchi R, Lorusso C, et al. Nutritional treatment of chronic liver failure. Hepatol Res. 2008;38(Suppl 1):S93-S101.

16. Khanna S, Gopalan S. Role of branched-chain amino acids in liver disease: the evidence for and against. Cur Opin Clin Nutr Metab Care. 2007;10(3):297-303. 
17. Capaldo B, Gastaldelli A, Antoniello S, et al. Splanchnic and leg substrate exchange after ingestion of natural mixed meal in humans. Diabetes. 1999;48(5):958-966.

18. Shimomura Y, Honda T, Shiraki M, et al. Branched-chain amino acids: catabolism in exercise and liver disease. J Nutr. 2006;136(1 Suppl):250S-253S.

19. Cascino A, Cangiano C, Calcaterra V, et al. Plasma amino acids imbalance in patients with liver disease. Am J Dig Dis. 1978;23(7):591-598.
20. Campollo O, Sprengers D, Dam G, et al. Protein tolerance to standard and high protein meals in patients with liver cirrhosis. World J Hepatol. 2017;9(14):667-676.

21. Christensen HN. Interorgan amino acid nutrition. Physiol Rev. 1982;62(4 Pt 1):1193-1233. 